\title{
An Open Source Power System Virtual Laboratory: The PSAT Case and Experience
}

\author{
Federico Milano, Member, IEEE, Luigi Vanfretti, Member, IEEE, and Juan Carlos Morataya, Member, IEEE
}

\begin{abstract}
This paper describes the authors' experience in the assessment of laboratory activities based on an open source software package for power system analysis, namely, Power System Analysis Toolbox (PSAT). PSAT is currently used in several universities for both undergraduate and graduate courses. PSAT has also its own Web forum, which provides support to students and researchers all around the world, thus resulting in an almost unique example of "virtual laboratory" over the Internet. This paper attempts to answer through a variety of real-life examples the following open questions: What are the practical and pedagogical advantages of using an open source software with respect to proprietary software for power system analysis? What happens if a power system virtual laboratory is freely available on the Web? What is the difference between a class-based and a Web-based virtual laboratory?
\end{abstract}

Index Terms-GNU Octave, Internet, Matlab, power system analysis, stability, time domain simulations, virtual laboratories.

\section{INTRODUCTION}

$\mathbf{E}$ LECTRIC power systems laboratories have always been "virtual" because practical experiences cannot be performed on a $\mathrm{HV}$ transmission system. In the past, Transient Network Analyzers (TNAs) were the only simulation tools available for research and education in electric power engineering [1]. However, the advent of digital analysis has led to a more convenient way of performing simulations through digital computers.

Computer-based simulations for power system analysis had wide success because electricity is invisible. Thus, reproducing real phenomena through computer-based simulations is an efficient solution for didactic and research purposes [2]. Power-flow analysis and short-circuit analysis have been for several years the basic tools of many power system courses [3]. In the last decade, inexpensive personal computers have made it possible to solve heavy time domain simulations, state estimation [4], optimal power flow, and reliability analyses. In recent years, the worldwide deregulation of electricity markets has strongly stimulated the investigation of complex mathematical programming

Manuscript received May 24, 2006; revised December 12, 2006. The work of Federico Milano was supported in part by the Ministry of Science and Education of Spain under CICYT Project DPI-2003-01362 and by Junta de Comunidades de Castilla-La Mancha under Project PBI-05-053.

F. Milano is with the Department of Electrical Engineering, University of Castilla-La Mancha, 13071 Ciudad Real, Spain (e-mail: Federico.Milano@uclm.es).

L. Vanfretti is with the Department of Electrical, Computer and Systems Engineering, Rensselaer Polytechnic Institute, Troy, NY 12181 USA (e-mail: vanfrl@rpi.edu).

J. C. Morataya is with the Planificación y Control, EEGSA-IBERDROLA, Guatemala, Guatemala (e-mail: JMorataya@eegsa.net).

Digital Object Identifier 10.1109/TE.2007.893354 problems involving innovative power network models and innovative market clearing procedures [5], [6].

Evidence of the importance of simulation in electric power engineering can also be seen in the variety of proprietary simulation tools currently available, the most widely-used being Power System Simulator for Engineering (PSS/E) by Power Technologies Inc. [7]. These tools represent a conventional choice for power system laboratories. However, commercial software is closed, i.e., does not allow changing the source code or adding new algorithms. For educational purposes, the flexibility and the ability of creating custom models are often more crucial aspects than computational efficiency. This paper discusses why an open source approach can be a valid alternative to commercial software for power system education.

The power system academic community has made admirable efforts to provide to students and researchers simulation tools that cover different aspects of electric power system analysis. Some examples of these software packages are UWPFLOW [8], PST [9], [10], PowerWeb [11], and ObjectStab [12]. A comprehensive list of power system software tools is available at [13].

This paper discusses the authors' experience in the assessment and development of a specific software package, namely, Power System Analysis Toolbox (PSAT) [14], which is currently used in several universities all around the world [15]. The authors' experience in the maintenance of a Web forum dedicated to PSAT is also presented in this paper.

PSAT is a free and open source because it is distributed free of charge and its source code can be modified by anyone [16]. This paper briefly discusses the difference between well-known open source broad-target projects (such as Apache or LaTeX) and an engineering software like PSAT.

The paper is organized as follows: Section II presents practical and pedagogical issues of setting up laboratory classes using PSAT. Section III describes the authors' experience in setting up class- and Web-based laboratories using PSAT. Finally, in Section IV, conclusions are drawn.

\section{SETTING UP A PSAT-BASED VIRTUAL LABORATORY}

PSAT is a Matlab-based toolbox for electric power system simulation and analysis. The command line version of PSAT is also GNU11 Octave compatible. PSAT includes power flow, continuation power flow, optimal power flow, small signal stability analysis, and time domain simulation. All operations can be assessed by means of graphical user interfaces; a SIMULINK-based library provides a user friendly tool for network design.

\footnotetext{
${ }^{1} \mathrm{GNU}$ is a recursive acronym that means "GNUs is Not Unix." Recursive acronyms are common in the open source community.
} 
A complete description of the software package PSAT is beyond the main scope of this paper. For interested readers, an in-depth description of all features of PSAT can be found in [14], [15]. This section focuses on practical and pedagogical issues associated with PSAT-based laboratories.

\section{A. Practical Issues}

The main practical components in setting up laboratory classes based on PSAT can be summarized as follows: system requirements, main goals, and assignment design.

Systems requirements are minimal. All one needs is an adequate number of personal computers where Matlab [17] or GNU Octave [18] are properly installed.

Setting up laboratory classes is also rather simple. PSAT comes with a set of sample test cases that can be used for didactic purposes. If modification or implementation of new features is necessary, the open source nature of PSAT makes these changes possible.

The assignments should be divided into stages of increasing complexity.

1) At the first stage the students should become familiar with the toolbox. Students need to understand the philosophy of the program (e.g., main features, graphical user interface, etc.).

2) The second stage is to become familiar with algorithms and data structures. This step will help to design specific assignments, such as the one illustrated in the Appendix.

3) For graduate courses or if the number of students is reduced, the students can be asked to implement new models and/or plug-ins.

In the authors' experience, a PSAT-based class leads to an increase of the students performance in several ways.

1) PSAT appears user-friendly to the students because it is based on Matlab, which typically has been used by students since their first year of undergraduate courses.

2) The students can familiarize themselves with the algorithms not only by using the program but also by reading the code.

3) The educator and the students can modify PSATs' algorithms and eventually add custom features.

4) PSAT runs on Linux and GNU Octave; therefore, no expensive license is needed to set up a laboratory using PSAT. This property is particularly relevant in developing countries where the students do not have access to proprietary software.

\section{B. Pedagogical Issues}

The current generation of students is accustomed to sophisticated software suites that aid academic work via the computer. However, in the authors' experience, the most serious drawback of proprietary software for educational purposes is the reduction of the freedom of the students. On the other hand, open source software while not limiting the user freedom tends to be less complete and intuitive than the proprietary type.

Thus, a compromise is needed. Open source software should have a reasonably user-friendly interface and should be written in a simple and high-level programming language (e.g., Matlab or GNU Octave).
In summary, the use of an open source package should be preferred for the following pedagogical issues.

1) The mind of the student should be opened. The student should not become accustomed to a program that gives all the answers.

2) The learning process should develop the curiosity of the student. Only if the code is open can the student explore all software features.

3) The students should understand that knowledge should be free and available to everyone.

\section{PSAT-BASED VIRTUAL LABORATORY EXPERIENCES}

This section discusses the authors' direct experience at the Universidad de Castilla-La Mancha (UCLM), Ciudad Real, Spain, and at the Universidad de San Carlos de Guatemala (USCG), Guatemala, Guatemala. Documented usage of PSAT in other universities and the usage of PSAT as a World Wide $W e b$ virtual laboratory are also illustrated in this section.

\section{A. The UCLM Case}

In Spain, undergraduate students of industrial engineering have to complete 375 credits ranging from basic courses (calculus, physics, chemistry, etc.) to elective courses of a specific area (mechanical, electrical, etc.).

A special course is the Project Laboratory, which is basically a one-term experience in a laboratory. The students contact the professor and get advised on a simple project. This project is typically used as the basis of the final one-year project that is needed for obtaining the degree.

At the undergraduate level, PSAT has been used in the laboratory segment of the course Advanced Electrical Machines (fourth year students) to illustrate the behavior of Automatic Voltage Regulators (AVRs) and turbine governors of synchronous machines. The students were asked to run several simulations of the 14-bus test system (see the Appendix) by changing the fault location and varying AVR and synchronous machine parameters.

For the Project Laboratory course, students are asked to familiarize themselves with power-flow analysis using PSAT. The project is divided into three assignments.

1) Setting up a test system data file (e.g., the IEEE 14-bus or 24-bus test system) and double-checking the power-flow solution with the one obtained using the sample data files provided with PSAT.

2) Solving a series of power-flow analyses by increasing load powers and considering generator reactive power limits, voltage limits, and current limits in transmission lines. The aim of this assignment is to reproduce the results of continuation power-flow analysis and to understand the concept of the maximum loading condition of a network.

3) Computing sensitivities of power-flow variables (e.g., voltages and generator reactive powers) with respect to network parameters (e.g., line reactances and shunt admittances) and load parameters (e.g., active and reactive powers).

So far, PSAT has been used as the main tool for one final project. The student was asked to write a routine similar to what has been proposed in [6] but using a Direct Current Optimal 
(a)

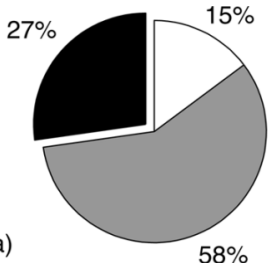

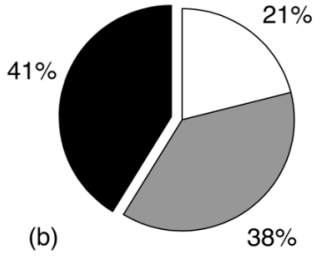

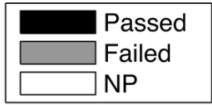

Fig. 1. Comparison between first and second semester of 2004 for the course Power System Analysis 1 at USCG. (a) First semester 2004. (b) Second semester 2004.

Power Flow (DC-OPF). The student was able to create his own routine quickly based on the existing PSAT code.

Finally, PSAT is used as a basic tool for the assignments of the Ph.D. course Angle, Frequency and Voltage Stability of Electric Power Systems. An example of a typical assignment is depicted in the Appendix.

Statistics for the UCLM are not relevant because of the reduced number of students. At the UCLM, the number of new students in the first year of industrial engineering is about 150 per year, but only about 15 of them will choose the power system specialization, and among those 15 , maybe only one is going to write code in Matlab for his final project. Nevertheless, to have a small number of students is also positive, because the students receive more attention from the professor and only highly motivated students are attending PSAT-based laboratory classes.

\section{B. The USCG Case}

Students of the electrical engineering program have to attend a five-year curriculum that includes two main parts; the first one provides basic courses (physics, mathematics, chemistry, etc.) while the second one offers specific power engineering courses.

PSAT has been used since 2004 in the Power System Analysis 1 course and is the main software tool of the recently-created power system laboratory.

Before the laboratory creation and because of the lack of funds at the University, the assignments of the Power System Analysis 1 course were performed using limited trial versions of a proprietary software package [19]. Clearly the trial constraints reduced the effectiveness of the assignments.

The creation of the laboratory was made possible by the donation of a few personal computers from the Student Association and former students. The teaching assistantships are conducted by volunteers (e.g., senior students). Finally, PSAT provided a free-of-charge educational tool.

The Power Systems Analysis 1 course is divided into several theoretical topics. Laboratory classes are scheduled a week after an adequate amount of topics have been covered in the theoretical classes. The learning process is verified by weekly laboratory assignments, two midterm exams, and a final exam. The laboratory activities are worth $15 \%$ of the total grade of the course. The minimum mark to pass the laboratory is $70 / 100$. Failing to pass the laboratory will result in also failing the whole course. PSAT-based classes have been given in 2004 and 2005 so far.

Year 2004: Fig. 1(a) and (b) shows the course results in 2004 with and without a PSAT-based laboratory, respectively.

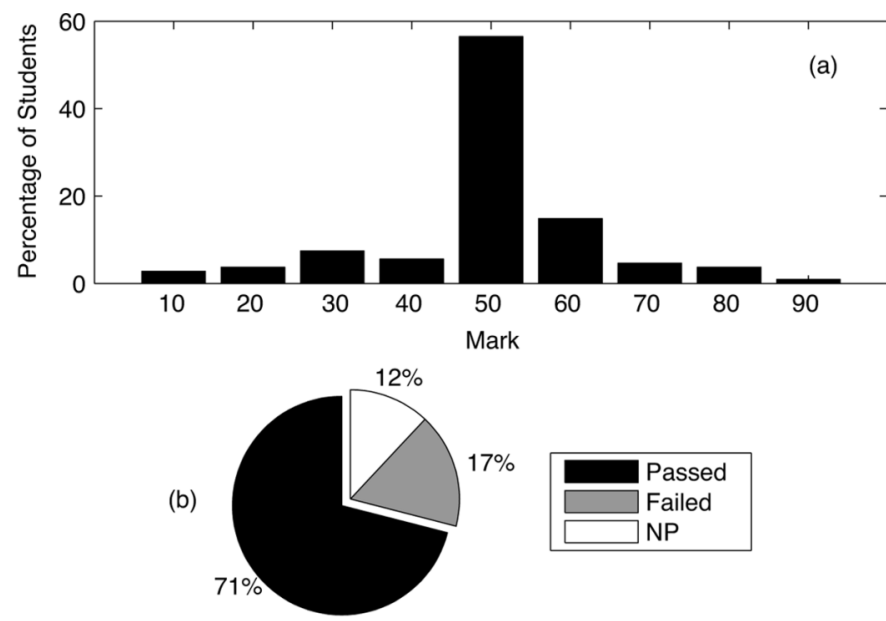

Fig. 2. Second semester 2004: PSAT-based laboratory class statistics. (a) Mark distribution. (b) Percentages of passed, failed, and "not presented" students.

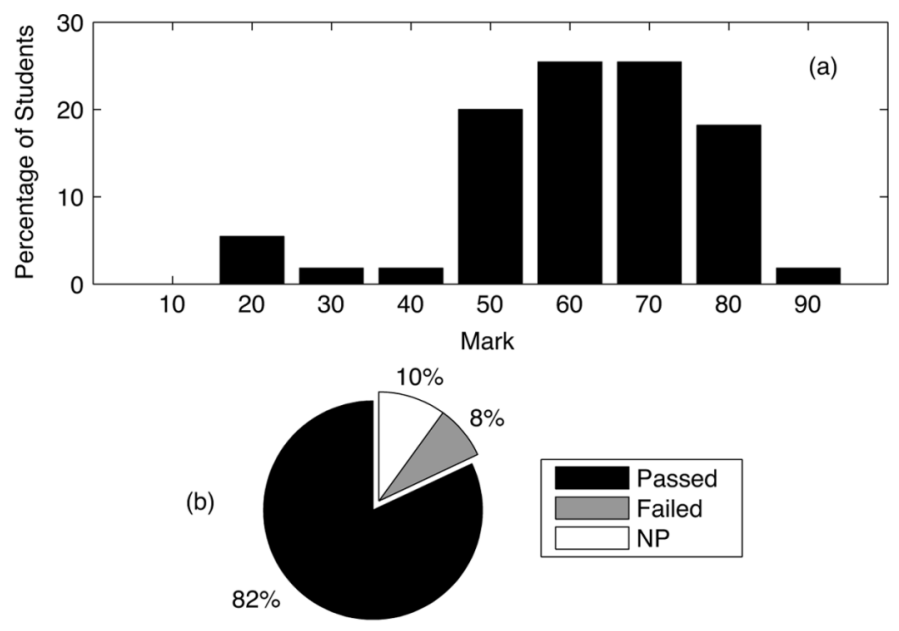

Fig. 3. Second semester 2005: PSAT-based laboratory class statistics. (a) Mark distribution. (b) Percentages of passed, failed and "not presented" students.

During the second semester the number of students that passed the course increased. This result is likely a result of the increased interest derived from laboratory experiences. Also the percentage of "not presented" (NP) students increases. When the laboratory was set up, a large number of students lacked computational skills and familiarity with Matlab and GNU Octave. Fig. 2(a) illustrates the marks obtained by the students for the 2004 laboratory activities. The total student number was 123 , and the average mark for the 2004 second semester was 73.5. Fig. 2(b) depicts the percentages of passed, failed, and "not presented" students.

Year 2005: Fig. 3(a) illustrates the marks obtained by the students for the 2005 laboratory activities. The total student number was 61 , and the average mark in the second semester 2005 was 77.1. Fig. 3(b) depicts the percentages of passed, failed, and "not presented" students. Observe that the laboratory activities of the second semester of 2005 were totally new, thus the students could not count on the results of 2004 assignments. In the second year after the creation of the PSAT-based laboratory, the mean mark increased, and the number of not presented students decreased. This result is relevant for USCG, because 
the percentage of not presented students is generally high (up to $75 \%$ ).

After attending Power System Analysis 1 laboratory classes, the students adopted PSAT as a standard tool for their activities. For example, a few students used PSAT in the course Project Evaluation and Planning and developed a project about reactive power compensation of a region of Guatemala. Other students used PSAT for their final project. For example, one of the projects currently in progress is about Automatic Generation Control (AGC) of power systems.

All in all, the PSAT-based laboratory classes have been a positive experience. Most students have completed the laboratory assignments with enthusiasm and, in some cases, provided useful suggestions to improve the quality of the classes.

\section{Other Cases}

PSAT is also used in the following universities as a teaching tool (see Appendix $\mathrm{J}$ of the PSAT documentation [15] for recommendation letters): University of Waterloo, Canada (see Appendix for an example of PSAT-based assignment proposed in this University); Universidad Mariano Gálvez de Guatemala; Universidad Campinas (Unicamp), Brazil; Universidad Centroamericana José Simeón Cañas, El Salvador; National Institute of Applied Sciences and Technology, Tunis; University of Maryland, USA; University of New South Wales, Australia; Asian Institute of Technology, Thailand; University of Kocaeli, Turkey; University of Genoa, Italy; Indian Institute of Technology, Kanpur, India; Federal University of Rio de Janeiro; and Indian Institute of Technology, Roorkee, India.

\section{Web-Based PSAT Laboratory}

Class-based PSAT laboratories have one common drawback - the number of students is typically reduced, ranging from less than 10 for Ph.D. courses to a few dozen for undergraduate courses or seminars. From a general point of view this situation should force one to think about the crisis of power system courses. From the point of view of the educator, the reduced number of students results in difficulty to get relevant feedback.

This situation can be drastically changed with a Web-based PSAT laboratory. By making the tool freely available on the Internet, any student interested in power-system analysis becomes a potential user of the program. This user will utilize the program for his own interests, laboratory activities or projects, thus checking the robustness of the program through random tests. In class-based PSAT laboratories, the educator guides the students so that they use PSAT in a conditioned way.

The Web-based experience of PSAT began in November 2002, when Prof. C. Cañizares, University of Waterloo, offered a Web page to Federico Milano (the Web page is available at http://www.power.uwaterloo.ca/ fmilano/). The idea was to share a free and "open source" Matlab-based software package for power-system analysis. Then, a public Web forum about PSAT was created on August 2003 on Yahoo. (The PSAT Forum is available at http://groups.yahoo.com/group/psatforum/.)

Fig. 4 illustrates the trend of the interest in the toolbox among the electric power system student community. This interest is measured in terms of PSAT-related mails received and sent per

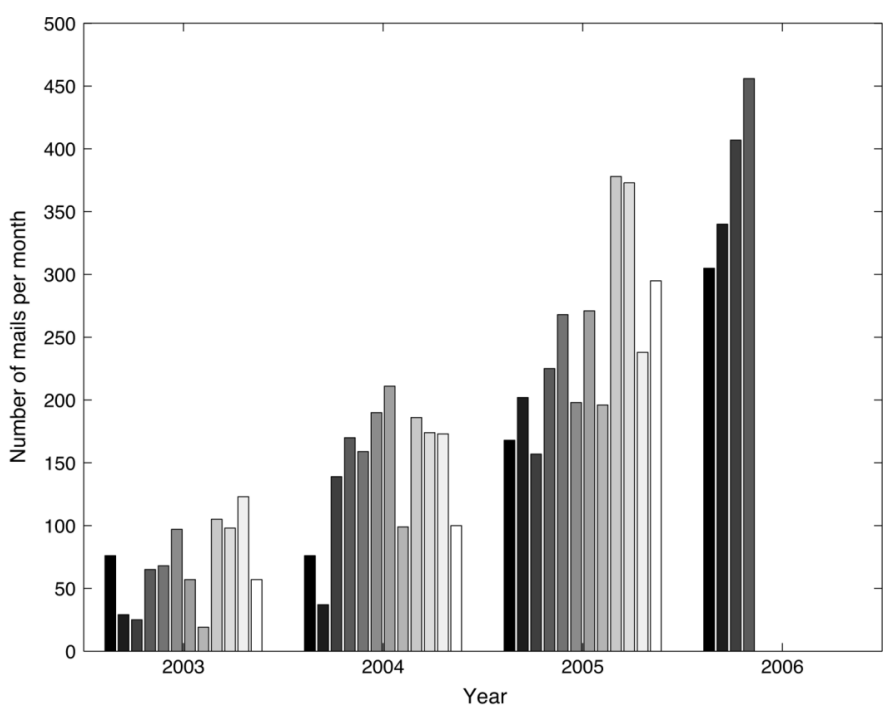

Fig. 4. PSAT-related emails per month.
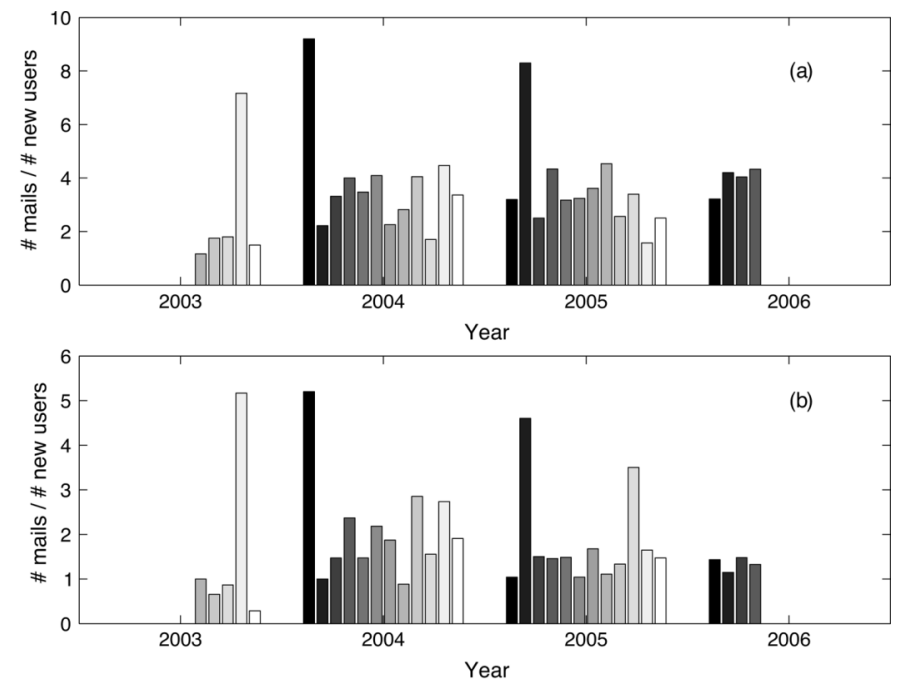

Fig. 5. Ratio of the number of emails per month and the number of new members of the PSAT Forum per month. (a) Total number of emails per month. (b) Number of emails per month related to errors or bugs of the program.

month. The rate of increase is rather constant and seems to be correlated with student term activities (e.g., the fall term).

In the experience of the maintainers of the PSAT Forum, new users typically tend to participate more actively than older ones. This is basically because of training issues and/or external constraints (e.g., the student has limited time to finish his project and is forced to solve quickly his problems). Observe also that new users are likely to solve new simulations that can lead to the discovery of undocumented bugs of the program.

Fig. 5(a) depicts the total number of new mails per month divided by the number of new members of the forum per month, while Fig. 5(b) depicts the number of mails per month concerning errors or bugs in the program divided by the numbers of new members of the forum per month. Bug reports do not necessarily correspond to real errors in the program since new users often input bad data to the program as a result of their lack of experience. 
One of the most interesting phenomena of typical open source software is that users feel involved in the development of the project. Instead of complaining about missing features or bugs, users often contribute suggestions, bug fixes, and even new code [16]. However, this situation has not been the case of PSAT. The miracle of a rapid growth and community-based development that is typical of many open source packages (for example, the Linux case) did not happen for PSAT. Based on the authors' experience, this is mainly a result of four causes.

1) The typical user of a program like PSAT is a student attending the last year of his or her undergraduate courses or at the beginning of his Master or Ph.D. However, this student is not yet experienced enough to write code by himself or herself.

2) Students attending the last years of their Master or Ph.D. courses are in principle the ideal candidates for contributing with new code. However, in this case, the student is more likely developing his or her own software tools and uses PSAT only as a benchmark or as a store from which to get ideas.

3) Researchers seldom use PSAT or contribute new code. This is a product of the conservativeness (i.e., closed mentality) of the scientific community that is not totally aware of the advantages of the "open source" way of thinking.

4) The users of a program like PSAT are a very reduced subset of the total number of university students and researchers. Thus, an open source project like PSAT cannot be compared with other open source packages, such as Apache or LaTeX, which are used by a broad range of people.

Although user contributions are not relevant, the emails that have been posted on the PSAT Web forum can be used as a database from which one can infer the student learning process.

1) All questions that the students post on the Web forum receive an answer. The questions can be either about the program features or power-system theory. The students also discuss power-system issues. Thus, the forum basically provides an online tutor to all students interested in power systems.

2) Several questions are raised when the results of a simulation are not as expected. The aim of the forum is to correct data errors, solve conceptual doubts, and guide proper understanding of simulation results. Furthermore, since the students are generally asked to share their data with the PSAT community, the forum also provides a database of case studies that is slowly but constantly increasing.

3) Some students seem to lack a supervisor (or their supervisor is not an expert on power systems), and the forum helps and guides these students.

Frequently asked questions (FAQs) give an idea of what students find more difficult and thus suggest what can be improved for making the toolbox more user-friendly. From the point of view of the software maintainer, the incidence of FAQs about missing models or algorithms can be used as a valuable database to understand which developments of PSAT could be more useful to the community and what is worth studying.

Table I depicts the incidence of relevant topics of powersystem analysis. Observe the interest on Flexible Alternating Current Transmission Systems (FACTS) devices, continuation
TABLE I

RELEVANT TOPICS AND THEIR INCIDENCE ON THE PSAT FORUM

\begin{tabular}{l|c}
\hline Topic & Incidence (\%) \\
\hline Slack bus & 16 \\
Infinite bus & 5 \\
Bifurcation theory & 4.5 \\
Continuation power flow & 28 \\
Optimal power flow & 32 \\
Small signal stability analysis & 10 \\
Time domain simulation & 23.5 \\
N-1 contingency analysis & 2.7 \\
Electricity markets & 5.8 \\
Wind Power & 3.8 \\
SVC & 22 \\
TCSC & 12 \\
STATCOM & 5.6 \\
SSSC & 10.5 \\
UPFC & 14.6
\end{tabular}

power flow, and optimal power flow (OPF) analyses. In many cases students ask for OPF methods with inclusion of FACTS devices, which is likely a current topic of Master and Ph.D. studies. Finally, observe the high occurrence of questions about slack bus and/or infinite bus, which is basically a symptom of the difficulties that students face in understanding these basic concepts.

\section{CONCLUSION}

The paper has presented the authors' experience with PSAT, an open source software package for teaching power-system analysis.

A variety of real-case PSAT-based virtual laboratories has been discussed in the paper. Examination results and Web forum activity are valuable databases for understanding most common issues of the students, the weaknesses of the program and what can be improved in the student learning process.

Future work will concentrate on improving PSAT reliability, and making it more intuitive. Also under development is the idea of setting up a Matlab server running PSAT to make the program available to everybody without the need of buying a Matlab license.

\section{APPENDIX}

This Appendix depicts a typical assignment that can be set up using PSAT. The assignment is proposed as part of the course E\&CE 664, Power System Computer Applications, offered by Prof. C. Cañizares at the University of Waterloo. A similar assignment is proposed as part of the Ph.D. course Angle, Frequency and Voltage stability of Electric Power Systems, offered by Prof. F. Milano at the University of Castilla - La Mancha.

\section{StABILITY ANALYSIS}

For the 14-bus IEEE benchmark system depicted in Fig. 6, and described in detail in the report: 


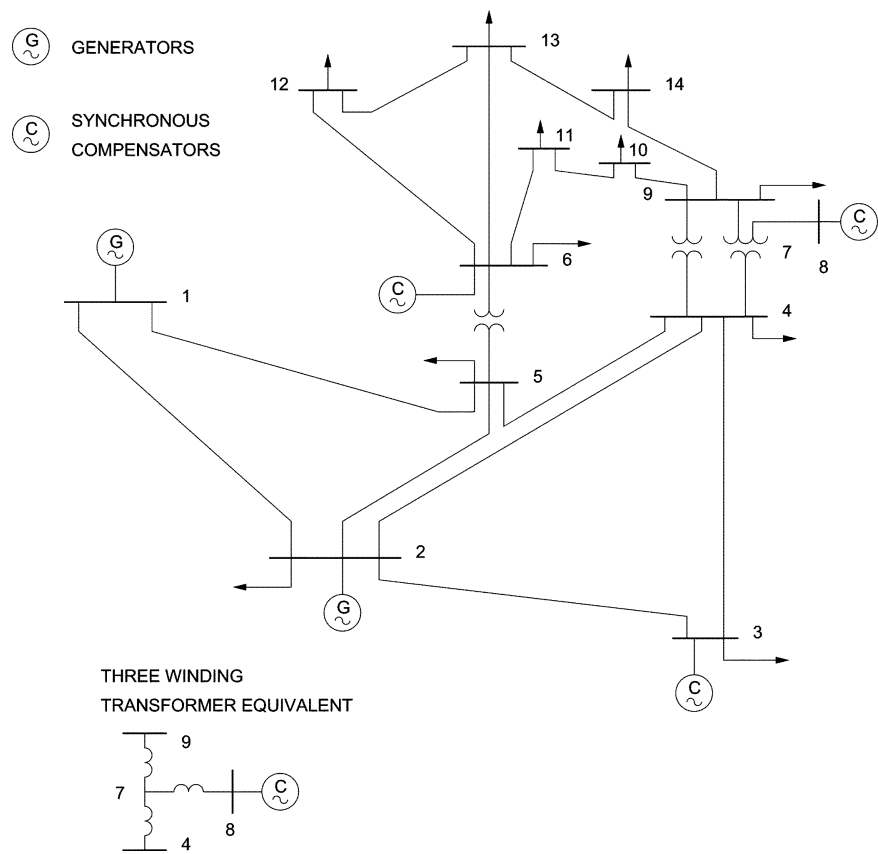

Fig. 6. IEEE 14-bus test system.

S. K. M. Kodsi and C. A. Cañizares, "Modeling and Simulation of IEEE 14-bus System with FACTS Controllers," Technical Report 2003-3, University of Waterloo, Waterloo, March 2003.

and using the simulation program PSAT, which is a Matlabbased program for power system analysis described in the paper:

F. Milano, "An Open Source Power System Analysis Toolbox," IEEE Transactions on Power Systems, vol. 20, no. 3, pp. 1199-1206, Aug. 2005.

as well as the PSAT data available at the PSAT Website.

1) Duplicate the Power/Voltage (PV) curves depicted below (extracted from the UW Report).

a) Obtain the PV curves by running a continuation power flow (CPF) using the base load and generation levels as the direction vector for the base system, and for the system without line 2-4 and without line 2-3, respectively. Make sure that you run PSAT using the following settings.

i) General Settings: Discard Dynamic Comp.; Use Distributed Slack Bus; Check PV reactive limits.

ii) CPF Settings: Qg limit controls; Initial Loading Param. $=1 ;$ Step Size Control = 0.1; no Single Slack Bus.

b) Compute the eigenvalues for the "upper" sides of the PV curves for all three cases, and show the existence of the Hopf bifurcations shown in this figure.

2) For a $40 \%$ loading increase $(\lambda=0.4)$, simulate a line $2-4$ trip in the time domain, and show that the system becomes unstable because of the existence of a Hopf bifurcation.

3) Demonstrate through eigenvalue analysis and time domain simulations that the system instability associated with the trip of line 2-4 for $\lambda=0.4$ can be removed by adding a PSS (Fig. 7) to the generator at Bus 1.

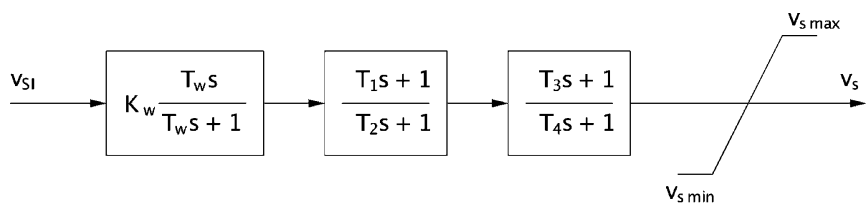

\begin{tabular}{|c|c|c|c|c|c|c|c|}
\hline $\mathrm{K}_{\mathrm{w}}$ & $\mathrm{T}_{\mathrm{w}}$ & $\mathrm{T}_{1}$ & $\mathrm{~T}_{2}$ & $\mathrm{~T}_{3}$ & $\mathrm{~T}_{4}$ & $\mathrm{~V}_{\mathrm{s}}^{\max }$ & $\mathrm{V}_{\mathrm{s}}^{\min }$ \\
\hline \hline 5 & 10 & 0.38 & 0.02 & 0.38 & 0.02 & 0.1 & -0.1 \\
\hline
\end{tabular}

Fig. 7. PSS scheme and data.

Choose the gain $K_{w}$ so that the system has a $2 \%$ damping ratio after the contingency, where the damping ratio for a complex eigenvalue $\mu=a+j b$ is defined as

$$
\zeta=\frac{a}{\sqrt{a^{2}+b^{2}}}
$$

\section{ACKNOWLEDGMENT}

The authors would like to thank Prof. C. Cañizares for his considerable help on the PSAT Web page and the information regarding course E\&CE 664 offered at the University of Waterloo. Thanks also to J. D. Alvarez Privado for his help and comments on the USCG case, and R. Smith for revising the English of this manuscript.

\section{REFERENCES}

[1] P. Anderson and A. Fouad, Power System Control and Stability. New York: Wiley, 2002.

[2] C. A. Cañizares and Z. T. Faur, "Advantages and disadvantages of using various computer tools in electrical engineering courses," IEEE Trans. Educ., vol. 40, no. 3, pp. 166-171, Aug. 1997.

[3] P. M. Anderson, Analysis of Faulted Power Systems. New York: Wiley, 1995.

[4] A. Abur, F. Magnago, and Y. Lu, "Educational toolbox for power system analysis," IEEE Comput. Appl. Power, vol. 13, no. 4, pp. 31-35, Oct. 2000.

[5] A. J. Conejo, F. Milano, and R. García-Bertrand, "Congestion management ensuring voltage stability," IEEE Trans. Power Syst., vol. 21, no. 1, pp. 357-364, Feb. 2006.

[6] F. Milano, C. A. Cañizares, and A. J. Conejo, "Sensitivity-based security-constrained OPF market clearing model," IEEE Trans. Power Syst., vol. 20, no. 4, pp. 2051-2060, Nov. 2005.

[7] Online Documentation PSS/E 30, Siemens Power Technologies Inc., Munich, Germany, Tech. Rep., 2004.

[8] C. A. Cañizares and F. Alvarado, UWPFLOW: Continuation and Direct Methods to Locate Fold Bifurcations in AC/DC/FACTS Power Systems, Apr. 2006 [Online]. Available: http://thunderbox.uwaterloo.ca/ $\sim$ claudio/software/pflow.htm

[9] J. H. Chow and K. W. Cheung, "A toolbox for power system dynamics and control engineering education and research," IEEE Trans. Power Syst., vol. 7, no. 4, pp. 1559-1564, Nov. 1992.

[10] G. Rogers and J. H. Chow, "Hands-on teaching of power system dynamics," IEEE Comput. Appl. Power, vol. 8, no. 1, pp. 12-16, Jan. 1995.

[11] R. D. Zimmerman and R. J. Thomas, "PowerWeb: A tool for evaluating economic and reliability impacts of electric power market designs," in Proc. IEEE Power Systems Conf. and Exposition, New York, Oct. 2004, pp. $1562-1567$.

[12] M. Larsson, "ObjectStab: An educational tool for power system stability studies," IEEE Trans. Power Syst., vol. 19, no. 1, pp. 56-63, Feb. 2004.

[13] Digital Educational Resources [Online]. Available: http://www.ece. mtu.edu/faculty/ljbohman/peec/Dig_Rsor.htm 
[14] F. Milano, "An open source power system analysis toolbox," IEEE Trans. Power Syst., vol. 20, no. 3, pp. 1199-1206, Aug. 2005.

[15] F. Milano, Power System Analysis Toolbox: Documentation for PSAT version 2.0.0, Mar. 2006 [Online]. Available: http://thunderbox.uwaterloo.ca/-fmilano

[16] R. M. Stallman, Free Software, Free Society: Selected Essays of Richard M. Stallman. Boston, MA: Free Software Foundation, 2002.

[17] Matlab Programming, 2006 [Online]. Available: http://www.mathworks.com

[18] J. W. Eaton, GNU Octave Manual. Bristol, U.K.: Network Theory Ltd., 1997.

[19] D. Glover and M. S. Sarma, Power System Analysis and Design. Chicago, IL: Thompson, 2002.

Federico Milano (M'03) received the Electrical Engineering and Ph.D. degrees from the University of Genoa, Genoa, Italy, in 1999 and 2003, respectively.

From September 2001 to December 2002, he was with the Electrical and Computer Engineering Department, University of Waterloo, Waterloo, ON, Canada, as a Visiting Scholar. He is currently an Assistant Professor of electrical engineering at Universidad de Castilla La Mancha, Ciudad Real, Spain. His research interests are voltage stability, electricity markets, and computer modeling of electric power systems.
Luigi Vanfretti (M'06) received the B.S. degree in electrical engineering from San Carlos University, Guatemala, Guatemala, in 2005.

He is currently a Master's student in the Electrical, Computer, and Systems Engineering Department at Rensselaer Polytechnic Institute, Troy, NY. His supervisor is Prof. Joe H. Chow. During fall 2005, he was a Visiting Academic in the Department of Electronics and Electrical Engineering at the University of Glasgow, Glasgow, Scotland, U.K. His research interests are dynamics, stability, control, and security of electric power systems.

Juan Carlos Morataya (M'06) received the B.Sc. in electrical power engineering at the University San Carlos of Guatemala, Guatemala, in 2004.

$\mathrm{He}$ is currently working at Empresa Eléctrica of Guatemala Iberdrola Group, Guatemala, Guatemala, as Transmission and Distribution Planning Engineer and as a Teacher of Electrical Engineering at the University San Carlos of Guatemala. His research interests are electrical machine modeling and voltage stability. 